\title{
ORIGINAL PAPER \\ COLON CANCER WITH MICROPAPILLARY CARCINOMA \\ COMPONENT: A CLINOPATHOLOGIC STUDY OF 9 CASES
}

\author{
Naoto Kuroda ${ }^{1}$, Kenji Yorita $^{1}$ \\ Department of Diagnostic Pathology, Kochi Red Cross Hospital, Kochi, Japan
}

\begin{abstract}
Recently, colon cancer with micropapillary pattern (MPP) has been identified. MPP is defined as tight tufts surrounded by cleft-like space and lacking true fibrovascular cores and showing reverse polarity. In this article, we studied nine cases of colon cancer with MPP. MPP usually accounted for a minor component in total tumour volume, which ranged from 3 to $40 \%$ with a mean percentage of $19.2 \%$. Associated histological subtype showed moderately differentiated tubular adenocarcinoma in all cases. The reverse polarity of villin ( $9 / 9$, diffuse) in MPP component was superior to that of CA125 (5/9, focal) or CD10 (5/9, diffuse 2/9, focal 3/9). In clinicopathological indicators such as sex, tumour location, tumour depth, lymphovascular invasion, lymph node metastasis, or pathological stage and clinical behaviour, there was statistically no difference between the MPP group and the non-MPP group of the colon. In conclusion, colon cancer with MPP is characterised by frequent association with moderately differentiated tubular adenocarcinoma as a minor component. Villin immunohistochemistry is useful in the detection of reverse polarity of MPP of colon cancer.
\end{abstract}

Key words: colon, micropapillary pattern, adenocarcinoma, villin.

\section{Introduction}

Carcinoma with micropapillary pattern (MPP) has been described in various organs including breast, urinary bladder, lung, ovary, and salivary gland $[1$, 2, 3, 4, 5, 6]. MPP is defined as tight small cell clusters surrounded by cleft-like space and lacking true fibrovascular cores and noting reverse polarity. Invasive micropapillary carcinoma arising in the colon has been for the first time been reported by Sakamoto et al. [7]. To date, the majority of reported cases are single case report $[8,9,10,11,12,13$, $14,15]$. However, there are only a few reports on a large scale clinicopathological study of colon cancer with MPP $[16,17,18,19,20]$. Although colon cancer with MPP behaves aggressively even in TNM Stage I and II, there is little evidence on the clinical outcome in this tumour [16]. Accordingly, in this article, we performed a clinicopathological study of nine cases of colon cancer with MPP and compared them with colon cancer without micropapillary pattern (non-MPP) in clinicopathological parameters and prognosis.

\section{Material and methods}

This study was approved by the Ethical Committee of Kochi Red Cross Hospital (No. 214). Among 617 surgically resected colon and rectal cancers between 2006 and 2016, 9 cases of colon cancer with MPP were extracted from pathological files. The sex ratio, age of patients, anatomic site of the tumour, gross findings, and pathological findings including percentage of MPP in total tumour volume, associated histological type, tumour depth, lymphovascular invasion, perineural invasion, lymph node metastasis and pathological stage, follow-up duration, additional therapy, and clinical outcome 
have been re-evaluated. Tumour stage was coded according to the seventh edition of the American Joint Committee on Cancer/Union for International Cancer Control staging system [21]. Histologic sections of surgically resected colon cancer were cut from formalin-fixed paraffin-embedded tissue blocks and stained with haematoxylin and eosin. For the immunohistochemistry, tissue sections were cut and stained with Ventana Benchmark Ultra autostainer (Ventana Medical Systems, Tucson, AZ). Primary antibodies against villin (ID2C3, 1 : 100, DAKO, Glostrup, Denmark), CD10 (56C6, prediluted, Novocastra Laboratories Ltd., Newcastle, UK), CA125 (M11, 1 : 50, DAKO, Glostrup, Denmark), E-cadherin (NCH-38, 1 : 100, DAKO, Glostrup, Denmark), vimentin (V9, 1 : 1600, DAKO, Glostrup, Denmark), HER2 (4B5, prediluted, Ventana, Tokyo, Japan), BRAFV600E (VE1, 1 : 50, Spring Biosciences, CA, USA), and ALK (5A4, prediluted, Nichirei, Tokyo, Japan) were employed in this study. For the comparison between colon cancer with and without MPP, 50 cases without MPP surgically resected in 2010 were selected. The sex (male or female), tumour site (right or left colon), tumour depth (pT1 or pT2 $\leq$ ), lymphatic invasion (absent or present), vascular invasion (absent or present), lymph node metastasis (absent or present), and pathological stage (low, less than II or high, more than III) were compared in both groups. Among 50 cases of non-MPP group, the clinical outcome of 38 cases were available. Comparison of clinicopathological parameters of both groups was performed by Fisher's exact probability test. Survival curves in both groups were constructed using Kaplan-Meier method, and the differences between curves were examined by long-rank test. A significant difference was considered for $\mathrm{p}$ values less than
0.05. In statistical analysis, BellCurve software for Windows (SSRI, Tokyo, Japan) was employed. Mutational analyses of $K-R A S$ and $N-R A S$ genes for one case were submitted to the laboratory of SRL company, Tokyo, Japan.

\section{Results}

\section{Clinical features}

Clinical characteristics are summarised in Table I. Colon cancer with MPP accounted for $1.5 \%$ (9/617) of all colon or rectal cancers. The patients consisted of 6 men and 3 women. One case has been previously reported [8]. The age of patients ranged from 51 to 79 years with a mean age of 65.6 years. Regarding the follow-up information, 6 cases were available. Two patients received adjuvant chemotherapy after the operation, and one patient underwent partial resection of the liver for subsequent liver metastasis. The follow-up duration ranged from 2 to 110 months with a mean of 52 months. Four patients were alive without disease and two patients were alive with distant metastasis.

\section{Pathological findings}

\section{Gross findings}

Macroscopic findings are summarised in Table II. Seven of nine tumours arose in the sigmoid colon. Five tumours showed ulcerative and localised type, and 4 tumours noted ulcerative and infiltrating type (Fig. 1). The cut surface showed a whitish colour with a various proportion of necrosis. No haemorrhage was seen in any of the cases. The size of tumour in the largest dimension ranged from 13 to $70 \mathrm{~mm}$ with a mean size of $49.4 \mathrm{~mm}$.

Table I. Clinical summary of colon cancer with micropapillary component

\begin{tabular}{|c|c|c|c|c|c|}
\hline Case & AGE & SEX & ADDITIONAL THERAPY & $\begin{array}{c}\text { FOLLOW-UP } \\
\text { (MONTHS) }\end{array}$ & Outсоме \\
\hline 1 & 70 & $\mathrm{~F}$ & None & 110 & AWOD \\
\hline 2 & 67 & $\mathrm{M}$ & None & 100 & AWOD \\
\hline 3 & 60 & $\mathrm{~F}$ & - & Lost & - \\
\hline 4 & 79 & $\mathrm{M}$ & $\begin{array}{c}\text { Resection for liver } \\
\text { metastasis } \\
(8 \text { months })\end{array}$ & 54 & AWOD \\
\hline 5 & 73 & $\mathrm{~F}$ & - & To another hospital & - \\
\hline 6 & 72 & $\mathrm{M}$ & Chemotherapy & 31 & AWD (LN) \\
\hline 7 & 51 & M & - & To another hospital & - \\
\hline 8 & 66 & $\mathrm{M}$ & Chemotherapy & 15 & AWD (liver) \\
\hline 9 & 52 & $\mathrm{M}$ & Chemotherapy & 2 & AWOD \\
\hline
\end{tabular}


Table II. Summary of gross findings of colon cancer with micropapillary component

\begin{tabular}{lccc}
\hline CASE & Site & Classification & Size (MM) \\
\hline 1 & Sigmoid & Ulcerative And Localised & $35 \times 27 \times 6$ \\
\hline 2 & Sigmoid & Ulcerative And Infiltrating & $38 \times 26 \times 10$ \\
\hline 3 & Sigmoid & Ulcerative And Infiltrating & $64 \times 52 \times 7$ \\
\hline 4 & Sigmoid & Ulcerative And Localised & $13 \times 13 \times 6$ \\
\hline 5 & Sigmoid & Ulcerative And Localised & $40 \times 25 \times 11$ \\
\hline 6 & Sigmoid & Ulcerative And Infiltrating & $70 \times 50 \times 10$ \\
\hline 8 & Descending & Ulcerative And Localised & $65 \times 48 \times 13$ \\
\hline 9 & Sigmoid & Ulcerative And Infiltrating & $50 \times 40 \times 13$ \\
\hline
\end{tabular}

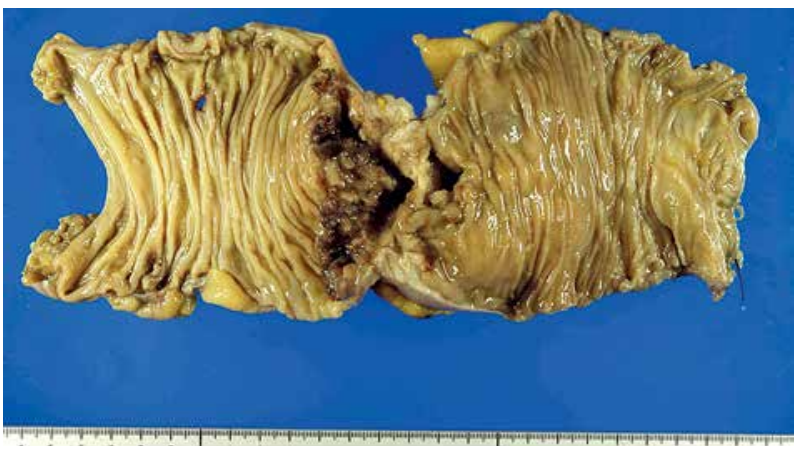

Fig. 1. Colon cancer with micropapillary carcinoma component usually shows ulcerative and localised type and is indistinguishable from usual adenocarcinoma

\section{Histological findings}

Histological features are summarised in Table III As shown as a definition, MPP consisted of small cell clusters surrounded by cleft-like space and lacking true fibrovascular cores and demonstrating reverse polarity, namely "inside-out" patterns. Tumour clusters of MPP were composed of approximately 3 to 20 cells. MPP usually accounted for a minor component in total tumour volume, which ranged from 3 to $40 \%$ with a mean percentage of $19.2 \%$ (Fig. 2A). Associated histological subtype showed moderately differentiated tubular adenocarcinoma in all cases (Fig. 2B). In addition, papillary adenocarcinoma and mucinous adenocarcinoma were identified in one case. The tumour depth consisted of 1 at the proper muscle, 3 at subserosa, 3 at serosa, and 2 in invasion to neighbouring tissue. Lymphatic and venous invasion by cancer cells was observed in 7 and 7 cases, respectively. Perineural invasion was noted in 4 cases. Metastasis to lymph node was noted in 6 cases. The pathological stage was composed of 3 cases in stage IV, 3 cases in stage IIIa, 2 cases in stage II, and 1 in stage I.

Table III. Summary of histological findings of colon cancer with micropapillary component

\begin{tabular}{|c|c|c|c|c|c|c|c|c|}
\hline Case & PMPP & OHT & TD & LYI & VI & PN & Node & Stage \\
\hline 1 & $40 \%$ & tub2, 60\% & SS & + & + & - & $\mathrm{pN1}$ & IIIa \\
\hline 2 & $5 \%$ & tub2, 95\% & SS & + & - & - & $\mathrm{pN} 1$ & IIIa \\
\hline 3 & $40 \%$ & tub2, $60 \%$ & SE & + & + & + & $\mathrm{pN} 1$ & IIIa \\
\hline 4 & $30 \%$ & tub2, $70 \%$ & $\mathrm{MP}$ & + & + & - & pNo & I \\
\hline 5 & $30 \%$ & tub2, $70 \%$ & SS & - & - & - & pN0 & II \\
\hline 6 & $10 \%$ & tub2, $90 \%$ & SI & + & + & + & $\mathrm{pN} 2$ & IV \\
\hline 7 & $5 \%$ & tub2, $95 \%$ & SE & + & + & + & $\mathrm{pN} 2$ & IV \\
\hline 8 & $10 \%$ & tub2, $90 \%$ & SE & + & + & + & $\mathrm{pN} 2$ & IV \\
\hline 9 & $3 \%$ & $\begin{array}{c}\text { Tub2, } 80 \% \\
\text { pap } 15 \% \\
\text { muc, } 2 \%\end{array}$ & SI & - & + & - & pN0 & II \\
\hline
\end{tabular}

PMPP - percentage of micropapillary pattern; OHT - other histological type, tub2 - tubular adenocarcinoma, moderately differentiated; pap - papillary adenocarcinoma; muc - mucinous adenocarcinoma, TD - tumour depth; MP - proper muscle; SS - subserosa, SE - serosa; SI -invasion into another tissue, LyI - lymphatic invasion; VI - vascular invasion; $P N$ - perineural invasion 

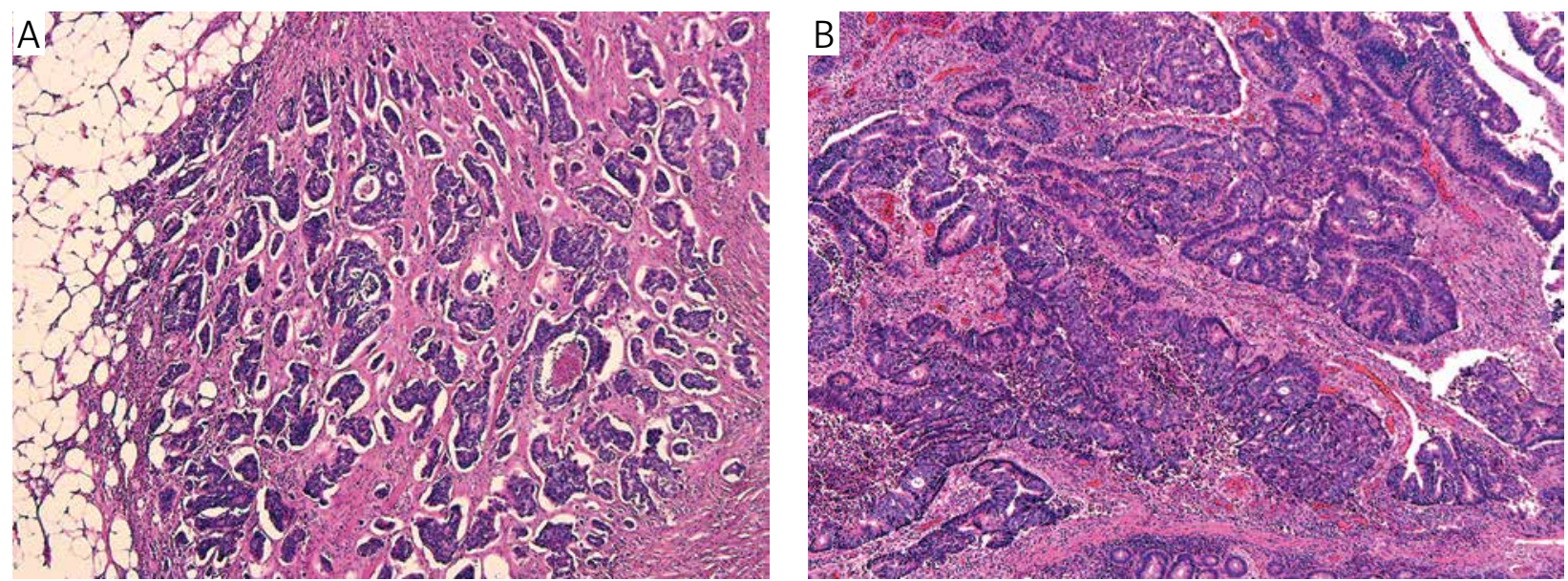

Fig. 2. A) Tumour clusters are usually small, consist of 3-20 tumour cells and have no fibrovascular cores. Vacant spaces between tumour clusters and stroma are observed (magnification $40 \times$ ). B) Associated histological subtype shows moderately differentiated tubular adenocarcinoma (magnification $40 \times$ )

\section{Immunohistochemical findings}

Immunohistochemical results of MPP are summarised in Table IV. Micropapillary carcinoma showed positivity for E-cadherin in all cases (Fig. 3A). When the expression of E-cadherin in MPP was compared with that of tubular adenocarcinoma, decreased expression of E-cadherin in membrane was observed in 7 of 9 tumours. Among them, four tumours showed aberrant cytoplasmic expression. No reactivity for vimentin was found in any of the tumours (Fig. 3B). The reverse polarity of villin (Fig. 3C) $(9 / 9$, diffuse $8 / 9$, focal $1 / 9)$ in the micropapillary carcinoma component was superior to that of CA125 (6/9, focal) or CD10 (5/9, diffuse 3/9, focal 2/9) (Fig. 3D). Neoplastic cells demonstrated no positive reaction for HER2, BRAFV600E, and ALK proteins in all tumours with MPP.

\section{Molecular genetic findings}

The mutation of codons $12,13,59,61,117$, and 146 in $K-R A S$ and $N-R A S$ genes was negative in one case (Case 6).

\section{Comparison of clinicopathological parameters and prognosis between micropapillary and non-micropapillary groups}

As shown in Table V, there were statistically no significant differences in sex, tumour site, tumour depth, lymphatic invasion, vascular invasion, lymph node metastasis, and pathological stage between colon cancer with MPP and non-MPP groups. Furthermore, there was no difference in overall and recurrence-free survival between both groups, as noted in Fig. 4.

\section{Discussion}

Gross finding of colon cancer with MPP seems to be indistinguishable from colon cancer without MPP. The propensity for sigmoid colon in MPP seems to reflect the original propensity of usual colon cancer. Histologically, micropapillary carcinoma is characterised by

Table IV. Immunohistochemical results

\begin{tabular}{|c|c|c|c|c|c|c|c|c|}
\hline CASE & E-CAD* & VIM & CA125 & Villin & CD10 & HER2 & $\begin{array}{c}\text { BRAF } \\
\text { V600E }\end{array}$ & ALK \\
\hline 1 &,+ dec & - & $\mathrm{F}+$ & $\mathrm{F}+$ & $\mathrm{F}+$ & - & - & - \\
\hline 2 &,+ dec & - & - & $\mathrm{D}+$ & - & - & - & - \\
\hline 3 &,+ dec & - & $\mathrm{F}+$ & $\mathrm{D}+$ & - & - & - & - \\
\hline 4 & + & - & - & $\mathrm{D}+$ & $\mathrm{D}+$ & - & - & - \\
\hline 5 & + & - & $\mathrm{F}+$ & $\mathrm{D}+$ & $\mathrm{D}+$ & - & - & - \\
\hline 6 &,$+ \mathrm{dec}$ & - & $\mathrm{F}+$ & $\mathrm{D}+$ & $\mathrm{F}+$ & - & - & - \\
\hline 7 & + , dec & - & $\mathrm{F}+$ & $\mathrm{D}+$ & - & - & - & - \\
\hline 8 & + , dec & - & $\mathrm{F}+$ & $\mathrm{D}+$ & $\mathrm{D}+$ & - & - & - \\
\hline 9 &,$+ \mathrm{dec}$ & - & - & $\mathrm{D}+$ & - & - & - & - \\
\hline
\end{tabular}

+ positive; - negative; $D$ - diffuse; $F$ - focal

*Expression of E-cadherin is compared with that of tubular adenocarcinoma. Dec-decreased expression. 

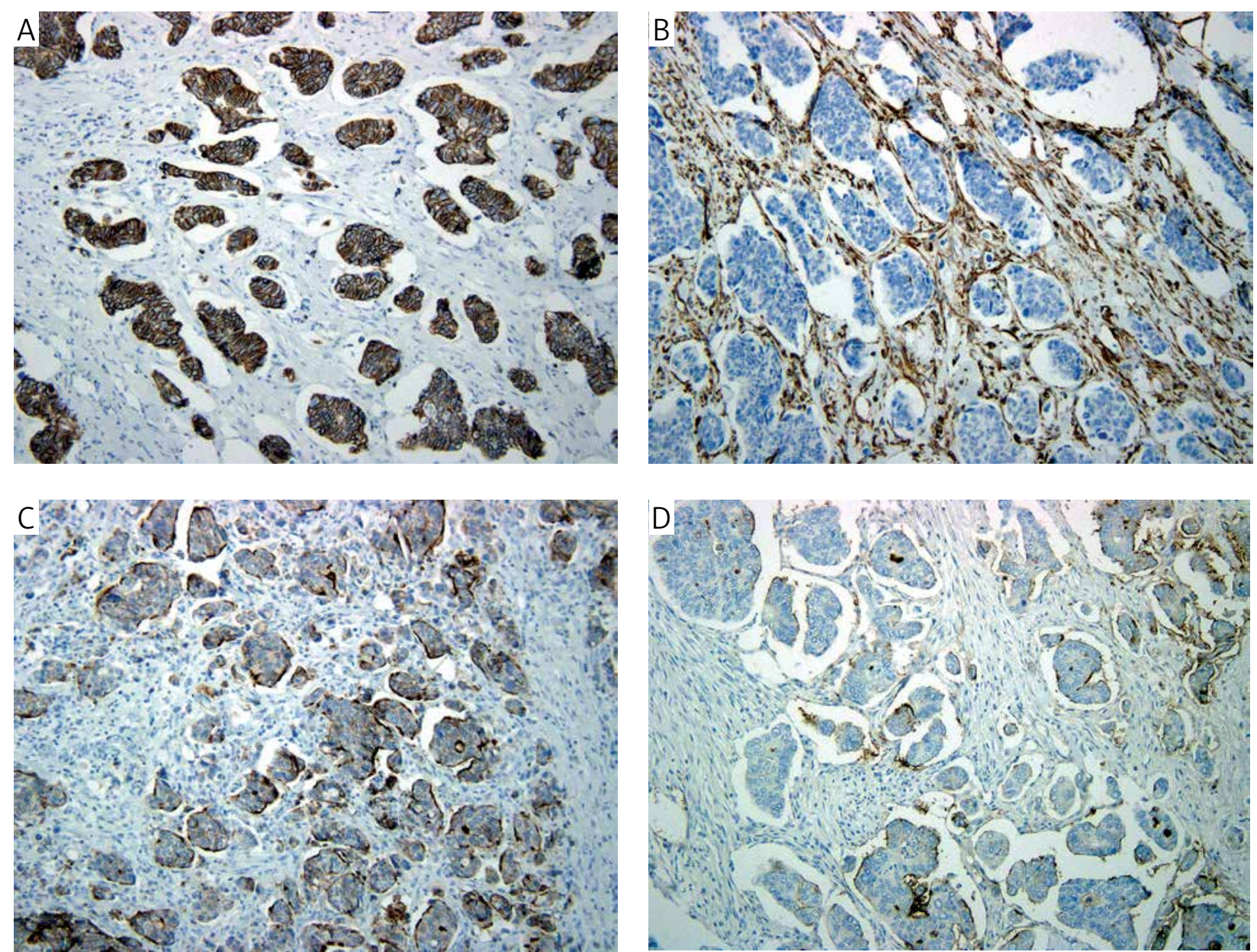

Fig. 3. A) E-cadherin is diffusely expressed in the cell membrane of neoplastic cells (magnification $100 \times$ ). B) Tumour cells show no cytoplasmic expression of vimentin (magnification $100 \times$ ). C) Villin evidently highlights the reverse polarity of micropapillary pattern (magnification $100 \times$ ). D) CD10 partly shows the inside-out pattern of neoplastic cells (magnification $100 \times)$

Table V. Comparison of clinicopathological parameters between colon cancer with micropapillary and non-micropapillary components

\begin{tabular}{|c|c|c|c|c|c|}
\hline & MP & $(\mathrm{N}=9)$ & NON-MP & $(\mathrm{N}=50)$ & P-VALUE \\
\hline \multirow[t]{2}{*}{ Sex } & Male & 6 & Male & 24 & \\
\hline & Female & 3 & Female & 26 & 0.2351 \\
\hline \multirow[t]{2}{*}{ Site } & Right & 1 & Right & 18 & \\
\hline & Left & 8 & Left & 32 & 0.183 \\
\hline \multirow[t]{2}{*}{ Depth } & p'T1 & 0 & pT1 & 5 & \\
\hline & $>\mathrm{pT} 2$ & 9 & $>\mathrm{pT} 2$ & 45 & 0.4232 \\
\hline \multirow[t]{2}{*}{ LyI } & Present & 7 & Present & 27 & \\
\hline & Absent & 2 & Absent & 23 & 0.1687 \\
\hline \multirow[t]{2}{*}{ VI } & Present & 7 & Present & 24 & \\
\hline & Absent & 2 & Absent & 26 & 0.0983 \\
\hline \multirow[t]{2}{*}{ LN meta } & Present & 6 & Present & 18 & \\
\hline & Absent & 3 & Absent & 32 & 0.1392 \\
\hline \multirow[t]{2}{*}{ Stage } & High & 6 & High & 19 & \\
\hline & Low & 3 & Low & 31 & 0.1089 \\
\hline
\end{tabular}

LyI - lymphatic invasion; VI - vascular invasion; $L N$ - lymph nodes 

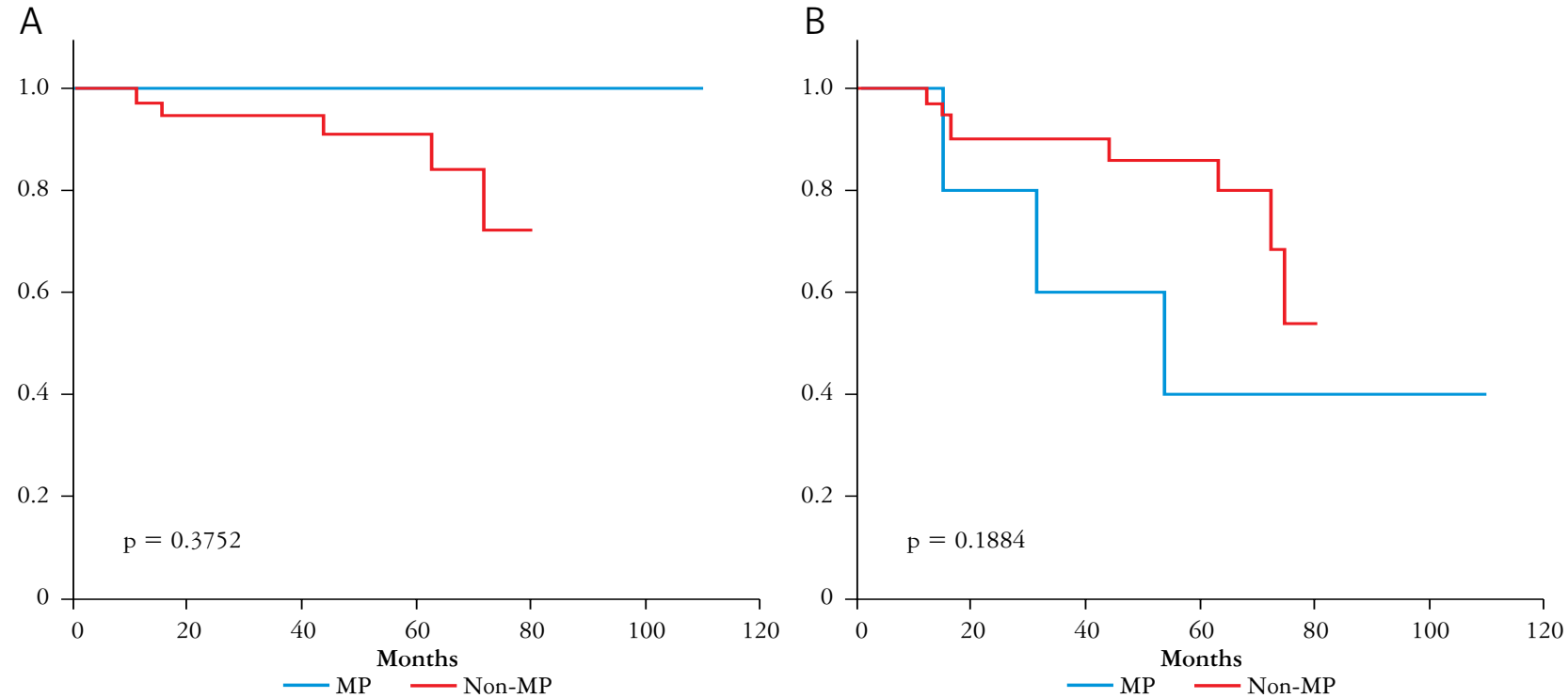

Fig. 4. The comparison of overall (A) and disease-free (B) survival times in micropapillary and non-micropapillary groups

cell clusters consisting of a small number of neoplastic cells, lack of fibrovascular cores, and "inside-out" growth pattern. The "inside-out" pattern can be detected by MUC1 or EMA immunohistochemistry in most breast and urinary bladder cancers. However, these proteins are not useful for the detection of reverse polarity of colon cancer with MPP [17]. Instead, villin and $\mathrm{CD} 10$ seems to be available markers of reverse polarity in colonic micropapillary carcinoma [17]. In the present study, villin seems to be superior in the detection of reverse polarity to CD10 or CA125. Micropapillary carcinoma was associated with moderately differentiated tubular adenocarcinoma in all cases. This result is consistent with previous reports [7, 20]. Additionally, the micropapillary component is not main, but minor of neoplastic components, whose mean average accounted for $19 \%$ of the total tumour volume. This result is also compatible with the previous result [15]. In the present study, lymphatic invasion, vascular invasion, perineural invasion, and metastasis to lymph nodes were observed in 7, 7, 5, and 6 cases, respectively. Additionally, in the present study, 8 cases showed the invasion deeper than subserosa, and 6 cases showed a high stage, including IIIa and IV. These results seem to be similar to those of the previous report that micropapillary carcinoma had a higher frequency of lymphovascular invasion, perineural invasion, higher depth of invasion, and more positive lymph nodes than usual adenocarcinoma [17]. In contrast, some superficial colon cancers with micropapillary carcinoma up to the submucosal layer in invasive depth have been reported. However, even such cases can cause a marked lymphovascular invasion or metastasis tolymph nodes $[9,11,13]$. Therefore, pathologists need to seek micropapillary carcinoma carefully in colonic biopsy materials. Regarding the prognosis, previous researchers described that colonic micropap- illary carcinoma behaves in an aggressive fashion $[16$, 18]. However, no patients died of disease with follow-up duration from 2 to 110 months with a mean of 52 months. Furthermore, there were no significant differences in overall and disease-free survival times between MPP and non-MPP groups of colon cancer in the present study. This result may reflect the original clinical behaviour of colon cancer or may not affect the clinical outcome because of the minor component of micropapillary carcinoma. Recently, bladder micropapillary carcinoma was classified as a luminal subtype, a favourable prognosis group in gene expression profile [22]. It is possible that micropapillary carcinoma of the colon may resemble that of the bladder at the molecular genetic level. Gonzalez et al. have recently reported that epithelial-mesenchymal transition is common in colon micropapillary carcinoma [23]. In contrast, micropapillary carcinoma does not seem to be involved in epithelial-mesenchymal transition, based on the results of this study. The alteration of BRAF or $A L K$ gene has been identified in colon cancer $[24,25,26]$. However, these genes do not seem to be involved in the pathogenesis of colon micropapillary carcinoma. The number of cases of colon cancer with MPP in the present study is too small to draw conclusions of the biological behaviour of this tumour. Because RAS mutational analyses were performed in only one case, these data are not relevant. Accordingly, further examination in a large-scale study will be necessary in order to elucidate the biological behaviour or clinicopathological characteristics of colon cancer with MPP.

MPP should be discriminated from tumour budding and poorly differentiated clusters (PDCs). Tumour budding is defined as single cells or small clusters of dedifferentiated tumour cells at the invasive front [27]. PDCs are composed of five or more cancer 
cells without glandular formation both at the invasive edge and within the tumour [28]. MPP generally consists of three to 20 cells without fibrovascular cores and with cleft-like space at the interface to the stroma. Three categories share the epithelial-mesenchymal transition, namely with decreased expression of E-cadherin [27, 28]. Therefore, these categories may share overlapping features in morphological and biological aspects. In the differential diagnosis, tumour budding consists of single cells or small clusters, whereas MPP and PDCs are composed of several cells or small to moderate clusters. The degree of cleft-like space may be an important diagnostic clue in discriminating MPP from PDCs [28].

In conclusion, colon cancer with MPP is characterised by frequent association with moderately differentiated tubular adenocarcinoma as a minor component. In clinicopathological indicators such as sex, tumour location, tumour depth, lymphovascular invasion, lymph node metastasis, or pathological stage and clinical behaviour, there seems to be no difference between MPP and non-MPP of the colon. Villin immunohistochemistry is useful in the detection of reverse polarity of MPP of colon cancer.

\section{The authors declare no conflicts of interest.}

\section{References}

1. Siriaunkgul S, Tavassoli FA. Invasive micropapillary carcinoma of the breast. Mod Pathol 1993; 6: 660-662.

2. Amin MB, Ro JY, El-Sharkaway T, et al. Micropapillary variant of transitional cell carcinoma of the urinary bladder. Am J Surg Pathol 1994; 18: 1224-1232.

3. Amin MB, Tamboli P, Merchant SH, et al. Micropapillary component in lung adenocarcinoma. A distinctive histologic features with possible prognostic significance. Am J Surg Pathol 2002; 26: 358-364.

4. Prat J, De Nictolis M. Serous borderline tumors of the ovary: a long-term follow-up study of 137 cases, including 18 with a micropapillary pattern and 20 with microinvasion. Am J Surg Pathol 2002; 26: 1111-1128.

5. Nagao T, Gaffy TA, Visscher DW, et al. Invasive micropapillary salivary duct carcinoma. A distinct histologic variant with biological significance. Am J Surg Pathol 2004; 228: 319-326.

6. Nassar H. Carcinomas with micropapillary morphology. Clinical significance and current concepts. Adv Anat Pathol 2004; 11: 297-303.

7. Sakamoto K, Watanabe M, Cruz CDL, et al. Primary invasive micropapillary carcinoma of the colon. Histopathology 2005; 47: 479-484.

8. Kuroda N, Oonishi K, Ohara M, et al. Invasive miropapillary carcinoma of the colon: an immunohistochemical study. Med Mol Mophol 2007; 40: 226-230.

9. Kondo T. Colon invasive micropapillary carcinoma arising in tubulovillous adenoma. Pol J Pathol 2008; 3: 183-185.

10. Wen P, Xu Y, Frankel WL, Shen R. Invasive micropapillary carcinoma of the sigmoid colon: distinct morphology and aggressive behavior. Int J Clin Exp Pathol 2008; 1: 457-460.

11. Sonoo H, Kameyama M, Inatugi N, et al. Pedunculated polyp of early sigmoid colon cancer with invasive micropapillary carcinoma. Jpn J Clin Oncol 2009; 39: 523-527.
12. Otsubo K, Kubo N, Nakashima N, et al. A juvenile case of pulmonary lymphangitic carcinomatosis caused by sigmoid colon cancer with a component of micropapillary carcinoma. Intern Med 2011; 50: 2361-2365.

13. Mukai S, Takakura Y, Egi H, et al. Submucosal invasive micropapillary carcinoma of the colon with massive lymph node metastases: A case report. Case Rep Oncol 2012; 5: 608-615.

14. Miyaoka Y, Fujiwara A, Kotani S, et al. Primary micropapillary carcinoma of the colon with submucosal invasion: A case report. Endoscopy International Open 2016; 04: E744-E747.

15. Haupt B, Ro JY, Schwartz MR, Shen SS. Colorectal adenocarcinoma with micropapillary pattern and its association with lymph node metastasis. Mod Pathol 2007; 20: 729-733.

16. Xu F, Xu J, Lou Z, et al. Micropapillary component in colorectal carcinoma is associated with lymph node metastasis in T1 and T2 Stages and decreased survival time in TNM stage I and II. Am J Surg Pathol 2009; 33: 1287-1292.

17. Verdu M, Roman R, Calvo M, et al. Clinicopathological and molecular characterization of colorectal micropapillary carcinoma. Mod Pathol 2011; 24: 729-738.

18. Lino-Silva LS, Salcedo-Hernandez RA, Caro-Sanchez CH. Colonic micropapillary carcinoma, a recently recognized subtype associated with histological adverse factors: clinicopathological analysis of 15 cases. Colorectal Disease 2012; 14: e567-e572.

19. Ohtsuki Y, Kuroda N, Umeoka T, et al. KL-6 is another useful marker in assessing a micropapillary pattern in carcinomas of the breast and urinary bladder, but not the colon. Med Mol Morphol 2009; 42: 123-127.

20. Guzinska-Ustymowicz K, Niewiarowska K, Prycznicz A. Invasive micropapillary carcinoma: A distinct type of adenocarcinomas in the gastrointestinal tract. World J Gastroenterol 2014; 20: 4597-4606.

21. Sobin L, Gospodarowicz M, Wittekind C. Colon and Rectum. In: TNM classification of malignant tumours. $7^{\text {th }} \mathrm{ed}$. Wiley-Blackwell, Geneva, Switzerland 2009; 100-105.

22. Guo CC, Dadhania V, Zhang L, et al. Gene expression profile of the clinically aggressive micropapillary variant of bladder cancer. Eur Urol 2016; 70: 611-620.

23. Gonzales RS, Huh WJ, Cates JM, et al. Micropapillary colorectal carcinoma: clinical, pathological and molecular properties, including evidence of epithelial-mesenchymal transition. Histopathology 2017; 70: 223-231.

24. Malapelle U, Pisapia P, Sgariglia R, et al. Less frequently mutated genes in colorectal cancer: evidence from next-generation sequencing of 653 routine cases. J Clin Pathol 2016; 69: 767-771.

25. Yakirevich E, Resnick MB, Mangrey S, et al. Oncogenic ALK fusion in rare and aggressive subtype of colorectal adenocarcinoma as a potential therapeutic target. Clin Cancer Res 2016; 22: 3831-3840.

26. Summers M, Smith CG, Maughan TS, et al. BRAF and NRAS locus specific variants have different coutcomes on survival to colorectal cancer. Clin Cancer Res 2017; 23: 2742-2749.

27.Zlobec I, Lugli A. Epithelial mesenchymal transition and tumor budding in aggressive colorectal cancer: tumor budding as oncotarget. Oncotarget 2010; 1: 651-661.

28. Barresi V, Branca G, Vitarelli E, Tuccari G. Micropapillary pattern and poorly differentiated clusters represent the same biological phenomenon in colorectal cancer. A proposal for a change in terminology. Am J Clin Pathol 2014; 142: 375-383.

\section{Address for correspondence}

Naoto Kuroda

Department of Diagnostic Pathology

Kochi Red Cross Hospital

Shin-honmachi 2-13-51, Kochi City

Kochi 780-8562, Japan

e-mail: kurochankochi@yahoo.co.jp 